\author{
Markers of inequality in self-rated health in \\ Brazilian adults according to sex
}

Marcadores de desigualdad en la autoevaluación
de salud de adultos en Brasil, según el sexo

Marcadores de desigualdad en la autoevaluación
de salud de adultos en Brasil, según el sexo

\section{Marcadores de desigualdade na autoavaliação
da saúde de adultos no Brasil, segundo o sexo \\ Marcadores de desigualdade na autoavaliação
da saúde de adultos no Brasil, segundo o sexo}

de salud de adultos en Brasil, según elsexo de salud de adultos en Brasil, según el sexo

\section{Resumo}

O objetivo deste estudo foi analisar a autoavaliação da saúde da população adulta brasileira, segundo os marcadores de desigualdade em saúde (cor ou raça, região de residência, escolaridade, renda domiciliar per capita e classe social), estratificada pelo sexo. Foram estudados 59.758 indivíduos com 18 anos ou mais de idade, que participaram da Pesquisa Nacional de Saúde de 2013, inquérito domiciliar de base populacional. A coleta de dados foi feita por entrevistas face a face e algumas medidas físicas. A autoavaliação da saúde foi analisada como positiva, regular e negativa. Usando-se a regressão logística multinomial, foram estimados odds ratio (OR) brutos e ajustados e os respectivos intervalos de 95\% de confiança (IC95\%). Foram calculados os percentuais de concordância e o valor de kappa para comparar os valores obtidos pelos modelos de regressão e os valores esperados. A prevalência da autoavaliação de saúde positiva na população total foi de $66,2 \%$, sendo $70 \%$ nos homens e 62,6\% nas mulheres. Na análise ajustada, as chances de avaliar pior sua saúde foram significativamente mais elevadas entre os individuos de menor renda domiciliar per capita, com pior nível de escolaridade, das classes sociais mais desfavorecidas, moradores das regiões Norte e Nordeste e que se autodeclararam pardos e pretos. Políticas públicas voltadas à promoção e à recuperação da saúde desses grupos sociais mais vulneráveis podem impactar na redução das iniquidades em saúde persistentes no Brasil.

Autoavaliação; Disparidades nos Níveis de Saúde; Condições Sociais; Inquéritos Epidemiológicos
Jailson Lopes de Sousa 1,2

Gizelton Pereira Alencar 2

José Leopoldo Ferreira Antunes 2

Zilda Pereira da Silva 2

doi: 10.1590/0102-311X00230318

\author{
Correspondência \\ J. L. Sousa \\ Instituto Brasileiro de Geografia e Estatística. \\ Rua Urussuí 93, São Paulo, SP 04542-050, Brasil. \\ jailson.l.sousa@ibge.gov.br \\ 1 Instituto Brasileiro de Geografia e Estatística, São Paulo, \\ Brasil. \\ 2 Faculdade de Saúde Pública, Universidade de São Paulo, \\ São Paulo, Brasil.
}




\section{Introdução}

As desigualdades em saúde são diferenças sistemáticas no estado de saúde entre grupos populacionais causadas pela exposição real aos determinantes sociais da saúde (DSS). Denominam-se iniquidades em saúde quando essas desigualdades, além de sistemáticas e relevantes, são também evitáveis, injustas e desnecessárias 1. Para o monitoramento dessas desigualdades e iniquidades, a Organização Mundial da Saúde (OMS) reforça que as informações de saúde de uma população devem ser analisadas segundo sexo, mínimo de dois marcadores sociais (escolaridade, renda, classe social ocupacional e outros), grupo étnico/raça, outro relevante estratificador social e, no mínimo, uma medida de unidade geográfica 2 .

Dentre os desfechos indicados pela OMS para o monitoramento das condições de saúde populacionais estão a autoavaliação da saúde 2 , medida multidimensional de avaliação global da saúde, com base nos aspectos objetivos e, principalmente, subjetivos de cada indivíduo ${ }^{3}$. A subjetividade reflete a percepção que o indivíduo constrói sobre várias dimensões (física, mental e social) de sua vida em termos de funcionamento, desempenho, capacidade, bem-estar e qualidade de vida ${ }^{4}$. Com base em uma pergunta simples e geral, o informante define sua saúde em uma escala de quatro ou cinco pontos, passando do pior até o melhor estado de saúde percebido. Nos estudos epidemiológicos, essa variável tem sido analisada de forma dicotômica, agregando a categoria regular ora à condição positiva, ora à negativa 3 . No entanto, essas opções não permitem aferir gradientes da autoavaliação nem tampouco comparar diretamente as frequências de condições opostas. Ademais, receia-se que a inclusão da categoria intermediária em um dos dois polos da autoavaliação possa subestimar a associação entre esse desfecho e os determinantes sociais.

Estudos mostram que mulheres 5,6,7, pessoas de idade mais avançada 6,8, aqueles que se autodeclaram não brancos 9,10, com pior escolaridade 7,11, pior renda 12,13 e de classes sociais menos privilegiadas 13,14 apresentam maior probabilidade de avaliar negativamente sua saúde. A literatura também destaca que a autoavaliação da saúde é determinada por variáveis de contexto individual e de aspectos dos micro, meso e macro ambientes 15 .

As condições socioeconômicas são os principais determinantes de saúde das populações. Escolaridade, renda e ocupação têm sido mais frequentemente utilizados para definir a posição socioeconômica dos indivíduos 13,16 . Os aspectos capturados por essas medidas e os caminhos pelos quais elas afetam a saúde são diferentes e, até certo ponto, complementares ${ }^{17}$. Estudar a classe social, confrontando-a com os marcadores usuais de posição social, pode contribuir para melhores descrição, previsão e interpretação das variações de saúde entre grupos sociais 18,19 . Observa-se, ainda, que são escassos os estudos de classe social estratificados por sexo 20 , podendo esta pesquisa trazer mais elementos sobre as iniquidades sociais em saúde entre homens e mulheres nas sociedades contemporâneas.

O objetivo principal deste trabalho foi analisar a autoavaliação da saúde da população adulta brasileira, segundo os marcadores de desigualdade em saúde, estratificada pelo sexo. Como objetivo secundário, buscou-se analisar o potencial explicativo da classe social sobre as desigualdades na autoavaliação da saúde quando comparado com a escolaridade e a renda domiciliar per capita do informante.

\section{Métodos}

\section{Desenho de estudo, fonte e coleta de dados}

Realizou-se um estudo transversal valendo-se dos dados da Pesquisa Nacional de Saúde (PNS) de 2013, inquérito domiciliar de base populacional, realizado pelo Instituto Brasileiro de Geografia e Estatística (IBGE) em parceria com o Ministério da Saúde. A PNS foi aprovada pela Comissão Nacional de Ética em Pesquisa, do Conselho Nacional de Saúde, em junho de 2013 (no 10853812.7.0000.0008) 21.

O trabalho de campo da PNS ocorreu entre agosto de 2013 e fevereiro de 2014, e a coleta de dados aconteceu mediante entrevista face a face e aferição de medidas físicas, com base em um plano amostral complexo envolvendo conglomerados com três estágios de seleção: setor censitário ou conjunto de setores censitários (unidades primárias de amostragem), domicílios particulares perma- 
nentes (unidades secundárias) e um morador com 18 ou mais anos de idade por domicílio (unidades terciárias) 21.

\section{População de estudo}

Foram incluídas neste trabalho informações sobre os moradores adultos selecionados na PNS 2013. Do total de 69.994 possíveis entrevistados, 60.202 (86\%) tiveram entrevista e medidas físicas realizadas (peso, altura, circunferência de cintura e pressão arterial) 21. A amostra final para este estudo compreendeu 59.758 indivíduos, sendo 25.818 homens e 33.940 mulheres, após a exclusão dos que não apresentavam informação para cor ou raça $(\mathrm{n}=3)$ e dos que apenas ajudavam, sem remuneração, no trabalho de algum membro do domicílio $(n=441)$, que não puderam ser classificados na tipologia de classe social usada nem considerados como pessoas fora da força de trabalho.

\section{Variáveis de estudo}

A seleção das variáveis de estudo baseou-se no modelo teórico proposto por Souza 22, à luz da teoria de Dahlgreen \& Whitehead, em que a autoavaliação da saúde é determinada por variáveis dispostas em camadas, envolvendo desde as características individuais, dos micro, meso e macrodeterminantes sociais. Mesmo sabendo-se que as condições sociais também produzem seus efeitos sobre a saúde, por meio dos recursos psicossociais e dos estilos de vida (efeito indireto) 23 , no modelo analítico deste estudo optou-se por controlar as variáveis que estivessem nesta linha de causalidade, aferindo-se, assim, o efeito direto dos determinantes estruturais sobre a autoavaliação da saúde, podendo comparar a força desta associação entre os marcadores de desigualdade estudados.

A autoavaliação de saúde foi considerada atendendo a seguinte questão: "Em geral, como o(a) senhor(a) avalia sua saúde?”, com cinco opções de respostas agregadas em positiva (incluindo as categorias "muito boa" e "boa"), regular e negativa ("ruim" e "muito ruim"), conforme estudos anteriores 24,25 .

Como marcadores de desigualdade em saúde foram utilizadas as seguintes variáveis: cor ou raça autodeclarada (branca, parda, preta, amarela e indígena); região de residência (Sul, Sudeste, Centro-oeste, Norte e Nordeste); nível de escolaridade completo (Superior, Médio, Fundamental e nenhum); renda domiciliar per capita em salários mínimos $(>6,>3$ a $6,>1$ a 3 e $\leq 1)$; e classe social. A renda domiciliar per capita resultou da soma dos rendimentos brutos mensais em dinheiro e produtos de todos os indivíduos do domicílio, dividida pelo número total de moradores do domicílio, sendo posteriormente categorizada em salários mínimos. O salário mínimo vigente no Brasil em 2013 era de $\mathrm{R} \$ 678,0026$

A classe social foi definida com base na tipologia proposta por Santos 27 para o Brasil, com base em informações da posição na ocupação e do grupo ocupacional dos indivíduos (segundo a Classificação de Ocupações para Pesquisas Domiciliares - COD), utilizando-se a renda individual como alternativa metodológica proposta pelo próprio autor para diferenciar algumas posições de classe entre si, suprindo a ausência de informações como número de empregados, propriedade do estabelecimento e tamanho da propriedade agrícola na PNS 2013. Essa tipologia contempla 15 posições de classe, podendo ser agregadas nos seguintes agrupamentos sociais: posições privilegiadas (capitalistas e fazendeiros, especialistas autônomos, gerentes, empregados especialistas e pequenos empregadores); controladores de ativos de menor valor (autônomos com ativos e autônomos agrícolas); classe trabalhadora não destituída (empregados qualificados, supervisores e trabalhadores típicos) e destituídos de ativos (trabalhadores elementares, autônomos precários, empregados domésticos, agrícolas precários e trabalhadores excedentes). Seguindo a proposta de Rose \& O'Reilly 28 , a categoria "pessoas fora da força de trabalho" foi adicionada, que compreende pessoas que não trabalhavam nem procuravam emprego no mês de referência da pesquisa 29.

Como variáveis que poderiam influenciar a associação entre a autoavaliação de saúde e os marcadores de desigualdade em saúde, utilizaram-se idade (18-29, 30-44, 45-59 e $\geq 60$ anos), situação do domicílio (urbano e rural), vivência com cônjuge ou companheiro(a) (sim e não), atividade física nos últimos três meses (sim e não), tabagismo (nunca fumou, ex-fumante e fumante), consumo abusivo de álcool (sim e não) e obesidade (sim e não), mensurada pelo índice de massa corporal (IMC) $\geq 30 \mathrm{~kg} / \mathrm{m}^{2}$, 
obtido pela divisão do peso corporal medido em quilogramas e pela altura ao quadrado medida em metros $\left(\mathrm{kg} / \mathrm{m}^{2}\right)$ 30. Foi considerado consumo abusivo de álcool a ingestão de quatro ou mais doses, no caso das mulheres, e cinco ou mais doses, no caso dos homens, em uma única ocasião dentro dos últimos 30 dias 30. No modelo teórico aqui utilizado, essas variáveis estão na linha causal entre a exposição e o desfecho. Suas inclusões na análise têm o intuito de avaliar se o efeito da posição social sobre a saúde realiza-se apenas por intermédio de diferenças comportamentais e condições sociodemográficas entre as classes ou se a associação persiste significante mesmo após o ajuste pelos fatores mais proximais.

\section{Análise de dados}

A análise foi realizada no software Stata versão 11.0 (http://www.stata.com), com o módulo survey, considerando-se todos os elementos que definem uma amostragem complexa 14,21, tais como estratificação, conglomeração, probabilidades desiguais de seleção e peso amostral para o morador adulto selecionado, com correção de não entrevista e calibração pela projeção da população. Foram calculadas as frequências absolutas e relativas de todas as variáveis analisadas.

Para avaliar a associação entre marcadores de desigualdade em saúde e autoavaliação da saúde foram calculados odds ratio (OR) e os respectivos intervalos de 95\% de confiança (IC95\%), por meio de modelos de regressão logística multinomial estratificados pelo sexo, pois a literatura aponta diferenciais importantes na percepção da saúde entre homens e mulheres 5,6,7. Para cada marcador considerado, foram construídos modelos independentes, brutos e ajustados às variáveis que mostraram confundir a associação principal investigada. Para esses modelos, foram excluídos os indivíduos que se autodeclararam amarelos e indígenas devido ao pequeno número de observações.

Para averiguar qual dos modelos explicaria melhor o desfecho em questão, foram calculados os porcentuais de concordância absoluta e o valor de kappa de Cohen, para comparar os valores obtidos pelos modelos e o esperado tão somente devido ao acaso 31. Os indivíduos foram classificados na classe modal em termos de probabilidades estimadas pelo modelo.

\section{Resultados}

Dentre a população estudada, a maior proporção era do sexo feminino, com idades entre 30-44 anos, de cor ou raça branca, residente no Sudeste brasileiro, com nenhum nível de escolaridade completo, renda familiar per capita de até um salário mínimo, que encontrava-se fora da força de trabalho, residia em área urbana, vivia com cônjuge ou companheiro(a), não praticava atividade física nos últimos três meses, não fazia uso abusivo de álcool nos últimos trinta dias e não era obesa (Tabela 1).

A autoavaliação da saúde na população total foi positiva para $66,2 \%$, regular para $28 \%$ e negativa para 5,8\% (dados não apresentados em tabela). Observaram-se essas mesmas proporções de 70,3\%, $25 \%$ e $4,8 \%$ para os homens e de $62,6 \%, 30,7 \%$ e $6,8 \%$ para as mulheres, respectivamente. Analisando a autoavaliação segundo as variáveis investigadas, as prevalências da saúde positiva nos homens foram sempre maiores do que nas mulheres, exceto nas categorias de mulheres com renda domiciliar per capita maior que seis salários mínimos e nos agrupamentos sociais de posições privilegiadas e de pessoas fora da força de trabalho (Tabela 1).

Na regressão bruta (Tabela 2), quando comparadas à autopercepção positiva, as chances de perceberem sua saúde como regular e negativa, entre homens e mulheres, foram maiores entre os que se autodeclararam pretos e pardos, residentes no Norte e Nordeste, e entre as pessoas fora da força de trabalho. Observou-se ainda um gradiente crescente da pior autoavaliação da saúde na medida em que pioravam a escolaridade e a renda domiciliar per capita.

Analisando cada marcador de desigualdade em saúde por vez, ajustados pelas variáveis de controle (Tabela 3), as chances de perceberem sua saúde como regular em comparação à positiva, entre homens e mulheres, foram maiores naqueles que se autodeclararam pardos e pretos, residentes nas regiões Norte e Nordeste, com nenhuma escolaridade completa, renda per capita de até um salário mínimo e que encontravam-se fora da força de trabalho e do grupo de destituídos de ativos. Em relação à autoavaliação negativa versus a positiva, para homens e mulheres, as chances foram maiores entre os 
Tabela 1

Caracterização da população de estudo ( $n=59.758)$, segundo variáveis investigadas e categorias da autoavaliação de saúde. Brasil, 2013.

\begin{tabular}{|c|c|c|c|c|c|}
\hline \multirow[t]{4}{*}{ Variáveis } & \multicolumn{5}{|c|}{ Percentual com peso amostral } \\
\hline & \multirow{3}{*}{$\begin{array}{c}\text { Amostra } \\
\text { total }\end{array}$} & \multicolumn{4}{|c|}{ Autoavaliação de saúde } \\
\hline & & \multicolumn{2}{|c|}{ Homens } & \multicolumn{2}{|c|}{ Mulheres } \\
\hline & & Positiva & Negativa & Positiva & Negativa \\
\hline \multicolumn{6}{|l|}{ Sexo } \\
\hline Masculino & 47,3 & 70,3 & 4,8 & - & - \\
\hline Feminino & 52,7 & - & - & 62,6 & 6,8 \\
\hline \multicolumn{6}{|l|}{ Idade (anos) } \\
\hline $18-29$ & 26,0 & 85,2 & 1,7 & 78,3 & 1,9 \\
\hline $30-44$ & 30,7 & 77,0 & 2,8 & 70,2 & 4,4 \\
\hline $45-59$ & 25,2 & 61,3 & 6,5 & 53,2 & 9,6 \\
\hline$\geq 60$ & 18,1 & 47,1 & 10,9 & 42,4 & 13,0 \\
\hline \multicolumn{6}{|l|}{ Cor ou raça (autodeclarada) } \\
\hline Branca & 47,5 & 73,7 & 3,8 & 67,4 & 5,5 \\
\hline Parda & 41,9 & 67,1 & 5,9 & 57,9 & 7,4 \\
\hline Preta & 9,2 & 66,7 & 5,0 & 58,1 & 9,8 \\
\hline Amarela & 0,9 & 81,0 & 3,8 & 68,1 & 7,2 \\
\hline Indígena & 0,4 & 61,2 & 9,6 & 48,2 & 13,2 \\
\hline \multicolumn{6}{|l|}{ Região de residência } \\
\hline Sul & 14,7 & 73,5 & 4,0 & 66,1 & 6,5 \\
\hline Sudeste & 44,0 & 75,6 & 3,5 & 67,8 & 5,2 \\
\hline Centro-oeste & 7,4 & 71,8 & 4,8 & 64,9 & 6,0 \\
\hline Norte & 7,4 & 62,0 & 5,9 & 57,5 & 7,7 \\
\hline Nordeste & 26,5 & 61,5 & 7,0 & 52.5 & 9,5 \\
\hline \multicolumn{6}{|l|}{ Nível de escolaridade (completo) } \\
\hline Superior & 12,8 & 86,0 & 0,9 & 82,8 & 1,7 \\
\hline Médio & 32,9 & 81,7 & 1,7 & 75,1 & 2,8 \\
\hline Fundamental & 15,5 & 74,4 & 2,6 & 62,6 & 4,2 \\
\hline Nenhum & 38,8 & 54,7 & 9,3 & 44,0 & 13,0 \\
\hline \multicolumn{6}{|l|}{ Renda domiciliar per capita (salários mínimos) * } \\
\hline$>6$ & 4,2 & 84,7 & 1,0 & 85,6 & 2,5 \\
\hline$>3-6$ & 8,0 & 83,2 & 1,9 & 79,9 & 2,3 \\
\hline$>1-3$ & 38,2 & 74,4 & 3,4 & 66,7 & 5,3 \\
\hline$\leq 1$ & 46,6 & 63,0 & 6,9 & 55,3 & 8,8 \\
\hline \multicolumn{6}{|l|}{ Classe social } \\
\hline Posições privilegiadas & 8,6 & 83,7 & 1,4 & 85,2 & 1,5 \\
\hline Controladores de ativos de menor valor & 6,3 & 73,8 & 3,3 & 68,6 & 4,7 \\
\hline Classe trabalhadora não destituída & 28,5 & 80,3 & 1,3 & 75,7 & 2,1 \\
\hline Destituídos de ativos & 21,4 & 64,6 & 5,5 & 60,9 & 5,7 \\
\hline Pessoas fora da força de trabalho & 35,3 & 51,4 & 11,9 & 53,5 & 10,3 \\
\hline \multicolumn{6}{|l|}{ Situação do domicílio } \\
\hline Urbano & 86,6 & 72,4 & 4,0 & 64,0 & 6,4 \\
\hline Rural & 13,4 & 58,1 & 9,3 & 51,8 & 9,5 \\
\hline \multicolumn{6}{|l|}{ Vive com cônjuge/companheiro(a) } \\
\hline Sim & 61,2 & 67,1 & 4,9 & 62,0 & 6,8 \\
\hline Não & 38,8 & 76,0 & 4,6 & 63,3 & 6,6 \\
\hline
\end{tabular}

(continua) 
Tabela 1 (continuação)

\begin{tabular}{|c|c|c|c|c|c|}
\hline \multirow[t]{4}{*}{ Variáveis } & \multicolumn{5}{|c|}{ Percentual com peso amostral } \\
\hline & \multirow{3}{*}{$\begin{array}{c}\text { Amostra } \\
\text { total }\end{array}$} & \multicolumn{4}{|c|}{ Autoavaliação de saúde } \\
\hline & & \multicolumn{2}{|c|}{ Homens } & \multicolumn{2}{|c|}{ Mulheres } \\
\hline & & Positiva & Negativa & Positiva & Negativa \\
\hline \multicolumn{6}{|c|}{ Atividade física nos últimos 3 meses } \\
\hline Sim & 31,6 & 81,7 & 1,4 & 71,6 & 3,2 \\
\hline Não & 68,4 & 63,6 & 6,8 & 59,2 & 8,1 \\
\hline \multicolumn{6}{|l|}{ Tabagismo } \\
\hline Nunca fumou & 67,8 & 76,5 & 3,2 & 65,5 & 5,5 \\
\hline Ex-fumante & 17,5 & 59,4 & 7,3 & 53,3 & 10,6 \\
\hline Fumante & 14,7 & 62,8 & 7,1 & 54,1 & 10,7 \\
\hline \multicolumn{6}{|c|}{ Consumo abusivo de álcool ** } \\
\hline Não & 86,3 & 68,6 & 5,4 & 61,7 & 7,0 \\
\hline Sim & 13,7 & 76,4 & 2,5 & 74,0 & 3,7 \\
\hline \multicolumn{6}{|l|}{ Obesidade *** } \\
\hline Não & 78,4 & 71,4 & 4,6 & 65,0 & 5,8 \\
\hline Sim & 21,6 & 64,6 & 5,8 & 55,7 & 9,4 \\
\hline
\end{tabular}

Nota: As prevalências da autoavaliação de saúde regular para homens e mulheres não foram apresentadas na tabela. * O salário mínimo vigente no Brasil, em 2013, era de R\$678,00 26;

** Número de doses de bebida alcoólica consumida em uma única ocasião nos últimos 30 dias - homens: 5 ou mais doses; mulheres: 4 ou mais doses 29;

$* * *$ Índice de massa corporal $\geq 30 \mathrm{~kg} / \mathrm{m}^{2} 29$.

\section{Tabela 2}

Odds ratios (OR) brutos e os respectivos intervalos de 95\% de confiança (IC95\%) para a associação entre posição socioeconômica e autoavaliação de saúde da população adulta. Brasil, 2013.

\section{Variáveis}

\section{Autoavaliação da saúde}

OR bruto (IC95\%)

Homens

Mulheres

Regular vs. positiva Negativa vs. positiva Regular vs. positiva Negativa vs. positiva

\begin{tabular}{|c|c|c|c|c|}
\hline \multicolumn{5}{|c|}{ Cor ou raça (autodeclarada) } \\
\hline Branca & 1,00 & 1,00 & 1,00 & 1,00 \\
\hline Parda & $1,32(1,19 ; 1,46)$ & $1,72(1,42 ; 2,08)$ & $1,49(1,36 ; 1,63)$ & $1,57(1,33 ; 1,85)$ \\
\hline Preta & $1,39(1,17 ; 1,66)$ & $1,46(1,06 ; 2,02)$ & $1,38(1,18 ; 1,61)$ & $2,06(1,60 ; 2,65)$ \\
\hline \multicolumn{5}{|c|}{ Região de residência } \\
\hline Sul & 1,00 & 1,00 & 1,00 & 1,00 \\
\hline Sudeste & $0,92(0,79 ; 1,07)$ & $0,85(0,63 ; 1,13)$ & $0,95(0,83 ; 1,09)$ & $0,77(0,60 ; 1,00)$ \\
\hline Centro-oeste & $1,08(0,91 ; 1,28)$ & $1,23(0,88 ; 1,69)$ & $1,08(0,93 ; 1,24)$ & $0,91(0,70 ; 1,19)$ \\
\hline Norte & $1,72(1,44 ; 2,05)$ & $1,72(1,23 ; 2,40)$ & $1,44(1,24 ; 1,67)$ & $1,33(1,01 ; 1,74)$ \\
\hline Nordeste & $1,69(1,47 ; 1,95)$ & $2,07(1,60 ; 2,68)$ & $1,72(1,51 ; 1,95)$ & $1,82(1,44 ; 2,29)$ \\
\hline \multicolumn{5}{|c|}{ Nível de escolaridade (completo) } \\
\hline Superior & 1,00 & 1,00 & 1,00 & 1,00 \\
\hline Médio & $1,35(1,09 ; 1,68)$ & $1,93(1,07 ; 3,46)$ & $1,59(1,34 ; 1,88)$ & $1,76(1,23 ; 2,52)$ \\
\hline Fundamental & $2,05(1,62 ; 2,60)$ & $3,28(1,84 ; 5,86)$ & $2,86(2,38 ; 3,44)$ & $3,17(2,19 ; 4,60)$ \\
\hline Nenhum & $4,34(3,54 ; 5,33)$ & $16,15(9,56 ; 27,30)$ & $5,26(4,45 ; 6,21)$ & $13,93(10,07 ; 19,26)$ \\
\hline
\end{tabular}

(continua) 
Tabela 2 (continuação)

Variáveis
Autoavaliação da saúde

OR bruto (IC95\%)

Homens

Mulheres

Regular vs. positiva Negativa vs. positiva Regular vs. positiva Negativa vs. positiva

\begin{tabular}{|c|c|c|c|c|}
\hline \multicolumn{5}{|l|}{ Renda domiciliar per capita (salários mínimos) * } \\
\hline$>6$ & 1,00 & 1,00 & 1,00 & 1,00 \\
\hline$>3-6$ & $1,05(0,72 ; 1,52)$ & $1,81(0,70 ; 4,69)$ & $1,64(1,21 ; 2,22)$ & $1,00(0,49 ; 2,00)$ \\
\hline$>1-3$ & $1,77(1,29 ; 2,43)$ & $3,78(1,62 ; 8,86)$ & $3,12(2,38 ; 4,08)$ & $2,65(1,45 ; 4,82)$ \\
\hline$\leq 1$ & $2,81(2,05 ; 3,85)$ & $9,14(3,93 ; 21,24)$ & $4,79(3,67 ; 6,26)$ & $5,37(2,97 ; 9,70)$ \\
\hline \multicolumn{5}{|l|}{ Classe social } \\
\hline Posições privilegiadas & 1,00 & 1,00 & 1,00 & 1,00 \\
\hline Controladores de ativos de menor valor & $1,72(1,33 ; 2,21)$ & $2,75(1,44 ; 5,24)$ & $2,52(1,77 ; 3,60)$ & $3,66(1,67 ; 8,03)$ \\
\hline Classe trabalhadora não destituída & $1,26(1,01 ; 1,58)$ & $0,97(0,53 ; 1,78)$ & $1,94(1,49 ; 2,51)$ & $1,65(0,87 ; 3,11)$ \\
\hline Destituídos de ativos & $2,56(2,03 ; 3,24)$ & $5,17(2,86 ; 9,35)$ & $3,60(2,79 ; 4,64)$ & $5,47(2,98 ; 10,02)$ \\
\hline Pessoas fora da força de trabalho & $3,97(3,14 ; 5,01)$ & $14,13(7,97 ; 25,06)$ & $4,46(3,48 ; 5,72)$ & $11,16(6,24 ; 19,98)$ \\
\hline
\end{tabular}

Nota: foram excluídos os indivíduos de cores ou raças amarela e indígena.

* O salário mínimo vigente no Brasil, em 2013, era de R\$678,00 26.

Tabela 3

Odds ratios (OR) ajustados e os respectivos intervalos de 95\% de confiança (IC95\%) para a associação entre posição socioeconômica e autoavaliação de saúde da população adulta estratificada por sexo. Brasil, 2013.

\begin{tabular}{|c|c|c|c|c|}
\hline \multirow[t]{3}{*}{ Variáveis } & \multicolumn{4}{|c|}{$\begin{array}{l}\text { Autoavaliação da saúde } \\
\text { OR ajustado * (IC95\%) }\end{array}$} \\
\hline & \multicolumn{2}{|c|}{ Homens } & \multicolumn{2}{|c|}{ Mulheres } \\
\hline & Regular vs. positiva & Negativa vs. positiva & Regular vs. positiva & Negativa vs. positiva \\
\hline \multicolumn{5}{|c|}{ Cor ou raça (autodeclarada) } \\
\hline Branca & 1,00 & 1,00 & 1,00 & 1,00 \\
\hline Parda & $1,48(1,33 ; 1,64)$ & $1,92(1,57 ; 2,35)$ & $1,65(1,50 ; 1,81)$ & $1,81(1,52 ; 2,15)$ \\
\hline Preta & $1,47(1,22 ; 1,77)$ & $1,50(1,07 ; 2,10)$ & $1,38(1,17 ; 1,63)$ & $2,05(1,57 ; 2,67)$ \\
\hline \multicolumn{5}{|c|}{ Região de residência } \\
\hline Sul & 1,00 & 1,00 & 1,00 & 1,00 \\
\hline Sudeste & $0,92(0,79 ; 1,08)$ & $0,88(0,66 ; 1,19)$ & $0,94(0,81 ; 1,08)$ & $0,75(0,57 ; 0,98)$ \\
\hline Centro-oeste & $1,24(1,04 ; 1,47)$ & $1,57(1,12 ; 2,20)$ & $1,19(1,02 ; 1,38)$ & $1,05(0,80 ; 1,38)$ \\
\hline Norte & $2,08(1,72 ; 2,52)$ & $2,19(1,55 ; 3,10)$ & $1,73(1,49 ; 2,02)$ & $1,78(1,35 ; 2,35)$ \\
\hline Nordeste & $1,92(1,66 ; 2,22)$ & $2,41(1,85 ; 3,15)$ & $1,89(1,65 ; 2,16)$ & $2,12(1,68 ; 2,68)$ \\
\hline \multicolumn{5}{|c|}{ Nível de escolaridade (completo) } \\
\hline Superior & 1,00 & 1,00 & 1,00 & 1,00 \\
\hline Médio & $1,69(1,35 ; 2,12)$ & $2,25(1,24 ; 4,06)$ & $1,76(1,49 ; 2,08)$ & $1,98(1,38 ; 2,85)$ \\
\hline Fundamental & $2,33(1,82 ; 2,98)$ & $3,17(1,74 ; 5,78)$ & $2,95(2,44 ; 3,56)$ & $3,06(2,08 ; 4,49)$ \\
\hline Nenhum & $3,22(2,58 ; 4,01)$ & $8,17(4,74 ; 14,06)$ & $3,86(3,24 ; 4,60)$ & $7,90(5,55 ; 11,24)$ \\
\hline \multicolumn{5}{|c|}{ Renda domiciliar per capita (salários mínimos) ** } \\
\hline$>6$ & 1,00 & 1,00 & 1,00 & 1,00 \\
\hline$>3-6$ & $1,20(0,81 ; 1,79)$ & $2,01(0,75 ; 5,33)$ & $1,74(1,26 ; 2,39)$ & $1,05(0,53 ; 2,06)$ \\
\hline$>1-3$ & $2,00(1,43 ; 2,80)$ & $3,63(1,51 ; 8,73)$ & $3,47(2,59 ; 4,65)$ & $2,81(1,56 ; 5,06)$ \\
\hline$\leq 1$ & $3,41(2,43 ; 4,79)$ & $9,17(3,80 ; 22,10)$ & $6,05(4,50 ; 8,14)$ & $6,68(3,70 ; 12,04)$ \\
\hline
\end{tabular}

(continua) 
Tabela 3 (continuação)

\begin{tabular}{|c|c|c|c|c|}
\hline \multirow[t]{3}{*}{ Variáveis } & \multicolumn{4}{|c|}{$\begin{array}{l}\text { Autoavaliação da saúde } \\
\text { OR ajustado * (IC95\%) }\end{array}$} \\
\hline & \multicolumn{2}{|c|}{ Homens } & \multicolumn{2}{|c|}{ Mulheres } \\
\hline & Regular vs. positiva & Negativa vs. positiva & Regular vs. positiva & Negativa vs. positiva \\
\hline \multicolumn{5}{|l|}{ Classe social } \\
\hline Posições privilegiadas & 1,00 & 1,00 & 1,00 & 1,00 \\
\hline Controladores de ativos de menor valor & $1,39(1,06 ; 1,83)$ & $1,71(0,89 ; 3,27)$ & $2,00(1,39 ; 2,89)$ & $2,48(1,13 ; 5,47)$ \\
\hline Classe trabalhadora não destituída & $1,48(1,18 ; 1,87)$ & $1,03(0,56 ; 1,88)$ & $2,14(1,64 ; 2,79)$ & $1,86(0,99 ; 3,50)$ \\
\hline Destituídos de ativos & $2,54(1,98 ; 3,26)$ & $3,96(2,17 ; 7,23)$ & $3,23(2,49 ; 4,20)$ & $4,25(2,32 ; 7,79)$ \\
\hline Pessoas fora da força de trabalho & $2,97(2,29 ; 3,84)$ & $8,33(4,59 ; 15,10)$ & $3,13(2,41 ; 4,05)$ & $6,47(3,55 ; 11,80)$ \\
\hline
\end{tabular}

Nota: foram excluídos os indivíduos de cores ou raças amarela e indígena.

* Ajustado pela idade, situação do domicílio, vive com cônjuge ou companheiro(a), atividade física nos últimos três meses, tabagismo, consumo abusivo de álcool e obesidade;

** O salário mínimo vigente no Brasil, em 2013, era de R\$678,00 26.

que se declararam pardos e pretos, residentes na Região Nordeste, com nenhuma escolaridade, renda per capita de até um salário mínimo e para as pessoas fora da força de trabalho.

Analisando o gap entre os sexos da associação de cada um dos marcadores de desigualdade com a autoavaliação da saúde negativa, percebe-se que a força de associação foi maior nos homens, quando comparados às mulheres, em relação à renda domiciliar per capita (37,3\% maior), à classe social (28,8\% maior), à região de residência (13,7\% maior) e à escolaridade (3,4\%), e a variável cor ou raça mostrou-se mais fortemente associada à pior autoavaliação da saúde entre as mulheres (10,4\% maior) (Tabela 3 ).

Inserindo nos modelos os indicadores clássicos de posição socioeconômica simultaneamente e ajustados pelas variáveis de controle, classe social, escolaridade e renda domiciliar per capita apresentaram redução da força de associação com a autoavaliação da saúde regular e negativa, quando da presença no modelo de dois ou três destes indicadores (Tabela 4). Essa redução foi maior entre as mulheres do que nos homens. Por exemplo: olhando o componente negativo da autoavaliação da saúde nos homens, a força de associação da classe social foi 47,1\% menor quando adicionada a variável escolaridade no modelo e 43,2\% menor quando adicionada a renda domiciliar per capita (Tabela 4), quando comparados com cada indicador de posição socioeconômica individualmente (Tabela 3). Dentre as mulheres, essa redução foi de $58,1 \%$ no modelo com classe social e escolaridade, e de 51,3\% no modelo com classe social e renda domiciliar per capita (Tabela 4).

Todos os modelos de regressão ajustados, tanto para homens quanto para mulheres, apresentaram resultados semelhantes para o percentual de concordância (e o valor de kappa) entre os modelos obtidos e os esperados tão somente devido ao acaso, variando de $65,95 \%(0,11)$ a $66,54 \%(1,15)$ entre os homens e de $65,9 \%(0,20)$ a $66,53 \%(0,22)$ nas mulheres.

\section{Discussão}

A maioria dos homens e das mulheres adultos brasileiros avaliou sua saúde de forma positiva, porém, há diferença entre os sexos na autopercepção da saúde, justificando, assim, a abordagem de gênero como a primeira das desigualdades para este e outros desfechos em saúde 5,6,14,32. Dentre os marcadores de desigualdade nos homens, renda domiciliar per capita, classe social e escolaridade estiveram mais fortemente associados à pior autoavaliação da saúde. Nas mulheres, essa ordem foi alterada, sendo maiores para escolaridade, renda domiciliar per capita e classe social. O papel de destaque da escolaridade na associação com a autoavaliação negativa entre as mulheres é reflexo da redução da força de associação da renda domiciliar per capita e da classe social, pois a razão de chances da escolaridade com a autopercepção negativa foi bem parecida entre os sexos. 
Tabela 4

Odds ratios (OR) ajustados e os respectivos intervalos de 95\% de confiança (IC95\%) das variáveis de posição socioeconômica entre si e autoavaliação de saúde da população adulta estratificada por sexo. Brasil, 2013.

\section{Variáveis}

Homens
Classe social
Posições privilegiadas
Controladores de ativos de menor valor
Classe trabalhadora não destituída
Destituídos de ativos
Pessoas fora da força de trabalho
Nível de escolaridade (completo)
Superior
Médio
Fundamental
Nenhum

Renda domiciliar per capita (salários mínimos) **

$>6$

$>3-6$

$>1-3$

$\leq 1$

\section{Mulheres}

Classe social

Posições privilegiadas

Controladores de ativos de menor valor

Classe trabalhadora não destituída

Destituídos de ativos

Pessoas fora da força de trabalho

Nível de escolaridade (completo)

Superior
Médio
Fundamental
Nenhum

Renda domiciliar per capita (salários mínimos) **

$>6$

$>3-6$

$>1-3$

$\leq 1$
Autoavaliação da saúde

Regular vs. positiva

OR ajustado * (IC95\%)

Modelo 2

Modelo 3

$\begin{array}{ccc}1,00 & 1,00 & 1,00 \\ 0,97(0,72 ; 1,30) & 1,13(0,85 ; 1,50) & 0,93(0,68 ; 1,25) \\ 1,08(0,85 ; 1,38) & 1,12(0,87 ; 1,44) & 0,97(0,75 ; 1,26) \\ 1,64(1,25 ; 2,12) & 1,69(1,29 ; 2,21) & 1,36(1,03 ; 1,81) \\ 2,00(1,52 ; 2,64) & 2,00(1,51 ; 2,64) & 1,66(1,25 ; 2,22) \\ 1,00 & - & 1,00 \\ 1,59(1,25 ; 2,00) & - & 1,41(1,10 ; 1,80) \\ 2,06(1,58 ; 2,68) & - & 1,73(1,32 ; 2,27) \\ 2,69(2,11 ; 3,41) & - & 2,18(1,68 ; 2,81) \\ - & 1,00 & 1,02(0,67 ; 1,54) \\ - & 1,7(0,76 ; 1,720) & 1,35(0,94 ; 1,95) \\ - & & \end{array}$

$2,59(1,79 ; 3,74) \quad 1,83(1,25 ; 2,69)$

$1,00 \quad 1,00 \quad 1,00$

$1,40(0,96 ; 2,03) \quad 1,56(1,08 ; 2,26) \quad 1,29(0,89 ; 1,88)$

$1,56(1,18 ; 2,05) \quad 1,45(1,11 ; 1,89) \quad 1,28(0,97 ; 1,68)$

$1,75(1,33 ; 2,30) \quad 1,81(1,39 ; 2,35) \quad 1,32(1,00 ; 1,74)$

$1,74(1,33 ; 2,28) \quad 1,77(1,36 ; 2,30) \quad 1,32(1,00 ; 1,73)$

$\begin{array}{lll}1,00 & - & 1,00\end{array}$

$1,51(1,26 ; 1,80) \quad$ - $\quad 1,27(1,06 ; 1,52)$

$2,44(2,01 ; 2,96) \quad$ - $\quad 1,90(1,56 ; 2,32)$

$3,16(2,63 ; 3,79) \quad$ - $\quad 2,33(1,93 ; 2,83)$

$\begin{array}{cc}1,00 & 1,00 \\ 1,64(1,19 ; 2,28) & 1,49(1,09 ; 2,05) \\ 3,04(2,27 ; 4,08) & 2,34(1,75 ; 3,12) \\ 4,90(3,63 ; 6,61) & 3,40(2,52 ; 4,58)\end{array}$

Negativa vs. positiva

OR ajustado * (IC95\%)

Modelo $1 \quad$ Modelo $2 \quad$ Modelo 3

\section{Homens}

Classe social

Posições privilegiadas

Controladores de ativos de menor valor

Classe trabalhadora não destituída

Destituídos de ativos

$-$

- $\quad 1,64(1,19 ; 2,28)$

$3,40(2,52 ; 4,58)$

Pessoas fora da força de trabalho

$\begin{array}{ccc}1,00 & 1,00 & 1,00 \\ 98(0,49 ; 1,96) & 1,27(0,66 ; 2,43) & 0,92(0,47 ; 1,81) \\ 64(0,34 ; 1,22) & 0,70(0,38 ; 1,28) & 0,55(0,29 ; 1,05) \\ 97(1,04 ; 3,73) & 2,26(1,22 ; 4,27) & 1,54(0,81 ; 2,92) \\ 41(2,34 ; 8,29) & 4,73(2,58 ; 8,67) & 3,40(1,81 ; 6,39)\end{array}$

(continua) 


\begin{tabular}{|c|c|c|c|}
\hline & \multicolumn{3}{|c|}{$\begin{array}{l}\text { Negativa vs. positiva } \\
\text { OR ajustado * (IC95\%) }\end{array}$} \\
\hline & Modelo 1 & Modelo 2 & Modelo 3 \\
\hline \multicolumn{4}{|l|}{ Nível de escolaridade (completo) } \\
\hline Superior & 1,00 & - & 1,00 \\
\hline Médio & $2,20(1,16 ; 4,16)$ & - & $1,86(0,97 ; 3,57)$ \\
\hline Fundamental & $2,68(1,39 ; 5,19)$ & - & $2,13(1,09 ; 4,16)$ \\
\hline Nenhum & $5,96(3,28 ; 10,84)$ & - & $4,49(2,42 ; 8,31)$ \\
\hline \multicolumn{4}{|c|}{ Renda domiciliar per capita (salários mínimos) ** } \\
\hline$>6$ & - & 1,00 & 1,00 \\
\hline$>3-6$ & - & $1,89(0,71 ; 4,98)$ & $1,57(0,59 ; 4,19)$ \\
\hline$>1-3$ & - & $3,04(1,28 ; 7,21)$ & $1,89(0,77 ; 4,62)$ \\
\hline$\leq 1$ & - & $5,41(2,27 ; 12,91)$ & $2,94(1,20 ; 7,20)$ \\
\hline \multicolumn{4}{|l|}{ Mulheres } \\
\hline \multicolumn{4}{|l|}{ Classe social } \\
\hline Posições privilegiadas & 1,00 & 1,00 & 1,00 \\
\hline Controladores de ativos de menor valor & $1,52(0,71 ; 3,29)$ & $1,84(0,85 ; 3,97)$ & $1,43(0,68 ; 3,02)$ \\
\hline Classe trabalhadora não destituída & $1,23(0,66 ; 2,27)$ & $1,17(0,64 ; 2,13)$ & $1,00(0,56 ; 1,81)$ \\
\hline Destituídos de ativos & $1,75(0,96 ; 3,16)$ & $2,06(1,15 ; 3,67)$ & $1,28(0,72 ; 2,26)$ \\
\hline Pessoas fora da força de trabalho & $2,71(1,51 ; 4,85)$ & $3,15(1,77 ; 5,60)$ & $1,96(1,12 ; 3,44)$ \\
\hline \multicolumn{4}{|l|}{ Nível de escolaridade (completo) } \\
\hline Superior & 1,00 & - & 1,00 \\
\hline Médio & $1,61(1,15 ; 2,25)$ & - & $1,35(0,97 ; 1,87)$ \\
\hline Fundamental & $2,28(1,58 ; 3,27)$ & - & $1,73(1,20 ; 2,50)$ \\
\hline Nenhum & $5,53(3,99 ; 7,66)$ & - & $3,93(2,82 ; 5,49)$ \\
\hline \multicolumn{4}{|c|}{ Renda domiciliar per capita (salários mínimos) ** } \\
\hline$>6$ & - & 1,00 & 1,00 \\
\hline$>3-6$ & - & $1,00(0,50 ; 2,00)$ & $0,84(0,42 ; 1,66)$ \\
\hline$>1-3$ & - & $2,48(1,39 ; 4,42)$ & $1,54(0,87 ; 2,72)$ \\
\hline$\leq 1$ & - & $4,95(2,78 ; 8,82)$ & $2,62(1,48 ; 4,64)$ \\
\hline
\end{tabular}

Nota: foram excluídos os indivíduos de cores ou raças amarela e indígena.

* Ajustado pela idade, situação do domicílio, vive com cônjuge ou companheiro(a), atividade física nos últimos três meses, tabagismo, consumo abusivo de álcool e obesidade;

** O salário mínimo vigente no Brasil, em 2013, era de R\$678,0026.

Os resultados deste estudo reforçam a evidência de que a autoavaliação negativa é mais frequente em mulheres do que em homens 5,6,7. Na análise estratificada por idade (dados não apresentados aqui), as mulheres mostraram maiores chances de avaliar negativamente sua saúde em todos os grupos de idade, quando comparadas aos homens. A diferença de gênero na autopercepção da saúde é consistente com o estudo de outras medidas de morbidade, portanto, não pode ser atribuída apenas a uma maior inclinação das mulheres em relatar doenças 33. Algumas questões ajudam a entender essa disparidade. Primeiro, o estilo de vida dos homens aumenta as chances deles desenvolverem doenças que levam à morte precoce; as mulheres sofrem mais de doenças não fatais e incapacitantes 34. Segundo, há menor contato dos homens com os serviços de saúde, já que eles resistem mais do que as mulheres em procurar ajuda, o que poderia ser considerado um aspecto de fragilidade no seu papel socialmente construído 35 . Terceiro, há desigualdades na posição socioeconômica entre os gêneros, com as mulheres geralmente possuindo menos acesso a mercados de trabalho, menor renda, condições de trabalho mais precárias, menor independência econômica e pensões mais baixas quando idosas 5,7 .

As prevalências da autoavaliação positiva segundo os sexos encontradas neste estudo foram semelhantes aos achados de outras investigações de base populacional com adultos, sendo de 71,5\% 
nos homens e 66,4\% nas mulheres no Brasil, em 2008 24, e 67,4\% entre os homens e 62,5\% entre as mulheres na China, em 2013 36. Outras pesquisas identificaram outras prevalências: 82,8\% entre os homens e $88 \%$ entre as mulheres na Suécia $37 ; 78,7 \%$ entre os homens trabalhadores e $64,4 \%$ entre as mulheres trabalhadoras no Chile 38; e 96,5\% entre os homens e 93,5\% entre as mulheres no Cazaquistão 39. Essas diferenças podem ser entendidas em relação aos aspectos metodológicos dos estudos, com pontos de corte diferentes na categorização da autoavaliação da saúde 20 , além do componente subjetivo envolvido na medida 4 .

A piora nos rendimentos domiciliares per capita aumentou as chances de os indivíduos avaliarem sua saúde como regular ou negativa. Evidências científicas são robustas em apontar que o rendimento acrescenta vantagens capazes de gerar ganhos imediatos e cumulativos, bem como indivíduos economicamente mais desfavorecidos tendem a ter menos recursos capacitadores e sofrer maiores impactos negativos na saúde 14,37. A renda é considerada a medida mais direta da posição socioeconômica, embora a desigualdade socioeconômica não possa ser resumida à desigualdade de renda, englobando também o desemprego, a educação e a exclusão social 40. Há uma associação robusta e positiva entre renda e longevidade dos indivíduos 32 .

Foi identificado um gradiente na autoavaliação da saúde na medida em que piorava a escolaridade. Sabe-se que educação está relacionada com capital humano, recursos psicossociais, condições de vida, estilos de vida e melhores condições de saúde 41, mostrando que indivíduos mais escolarizados têm melhor saúde autorreferida 11,37 e menores taxas de morbidade e mortalidade 7 . Ainda não está claro na literatura se os benefícios da educação diferem entre homens e mulheres. Alguns resultados apontam maiores ganhos às mulheres 42,43 , outros apontam direções opostas 44 , e há os que não relatam nenhuma diferença estatisticamente significante 45,46. Duas hipóteses são levantadas para entender resultados divergentes: uma diz que devido as mulheres terem menos recursos socioeconômicos (menor independência econômica, menos oportunidade de emprego em tempo integral, menor autoridade no trabalho e outros) sua saúde seria mais favorecida como resultado do melhor desempenho educacional, reduzindo, assim, o efeito negativo da insuficiência dos outros recursos; outra hipótese explica que a saúde dos homens seria mais favorecida, pois o ganho no desempenho educacional ampliaria as vantagens socioeconômicas já presentes entre eles, quando comparados com as mulheres 42 .

Indivíduos fora da força de trabalho e aqueles pertencentes aos grupos sociais destituídos de ativos, que incluem trabalhadores elementares, autônomos precários, empregados domésticos, trabalhadores agrícolas e trabalhadores excedentes, apresentaram maiores chances de avaliarem sua saúde como regular ou negativa, tanto os homens quanto as mulheres. Esses resultados apontam que a exclusão do mercado de trabalho ou a execução de atividades profissionais de natureza extenuante, cansativa e arriscada, assim como a ausência de regulamentação dos contratos de trabalho, têm efeitos deletérios para a saúde 14 .

A literatura reconhece que a posição de classe além de definir muitos aspectos de vida dos indivíduos, também tem papel decisivo na modulação de outras desigualdades em saúde, tais como as étnicas e as de gênero 16, reforçando sua utilização em trabalhos sobre desigualdades em saúde. Estudos mostram que independentemente de raça, gênero e localização geográfica, os indivíduos das classes sociais desfavorecidas têm pior saúde e maior mortalidade do que os das classes privilegiadas 14,15,18. Ademais, o presente estudo mostrou que a influência da classe social sobre a autoavaliação da saúde continuou significante mesmo após ajuste por variáveis mais proximais no encadeamento causal do modelo de Dahlgreen \& Whitehead. Isso é verificável tanto para medidas objetivas de classe social, avaliadas neste trabalho pela combinação de renda, escolaridade e status ocupacional, quanto para medidas subjetivas, geralmente avaliadas pela comparação com recursos materiais de outros 16 .

Numa revisão sistemática, Moor et al. 23 estudaram a determinação da saúde autorreferida, comparando as vias materialista, psicossocial e comportamental, que explicam esta determinação. Os autores apontaram evidências de que as condições materiais e estruturais são mais importantes para as desigualdades sociais na autoavaliação da saúde do que os fatores psicossociais e comportamentais, e que seu efeito direto sobre a autoavaliação da saúde é maior do que seu efeito indireto, quando as características psicossociais e comportamentais estão na linha de causalidade.

A diminuição da força da associação de classe social, escolaridade e renda com a pior autoavaliação da saúde, quando da presença de duas ou de todas elas no modelo de regressão, demonstra 
a relação estreita e de colinearidade entre estas variáveis na determinação das condições de vida e de saúde 16,40, pois parte do efeito da classe social é mediada pela escolaridade e pela renda, já que a escolaridade está associada à posição ocupacional, que por sua vez está associada à renda 14. Porém, o efeito de uma variável não anula as demais, demonstrando que os aspectos capturados por essas medidas e os mecanismos pelos quais elas afetam a saúde são provavelmente diferentes 17. Os agrupamentos sociais derivados da tipologia de classe social de Figueiredo Santos 27 estiveram adequados para capturar desigualdades na autoavaliação da saúde da população adulta brasileira segundo sua posição social, demonstrando que o constructo de classe social responde por mecanismos que afetam o poder social, as chances de vida e as condições de saúde, que não são capturados pela escolaridade e pela renda 14,47 .

Embora em menor magnitude, as diferenças sociais relacionadas à cor ou raça e à macrorregião de residência refletem desigualdades na autoavaliação da saúde em ambos os sexos, reforçando a importância destes marcadores para o monitoramento das iniquidades em saúde 2 .

Indivíduos que se autodeclararam pretos e pardos avaliaram pior sua saúde entre homens e mulheres. No Brasil, há evidências de um contraste marcante de recompensas materiais entre brancos e negros (pretos e pardos), colocando os últimos em posições inferiores na hierarquia social, com reflexos em uma desvantagem histórica que afeta a trajetória de vida destas pessoas e seus estados de saúde 48 .

As chances de uma pior autoavaliação da saúde foram maiores entre os moradores do Norte e do Nordeste do Brasil. As pessoas são afetadas pelos locais de residência, pois as implicações para a saúde são decorrentes da composição de fatores protetivos e do sistema de oportunidades associados ao ambiente físico, características socioculturais e históricas dos lugares 49 . A existência de uma enorme desigualdade social entre as regiões do país, marcada por uma heterogeneidade regional que persiste ao longo da história, constitui dois blocos bem distintos entre Norte/Nordeste e Sul/Sudeste/ Centro-oeste ${ }^{49}$, com as primeiras regiões caracterizadas historicamente por maiores desigualdades internas, menor renda média, condições de vida precárias e disparidades na distribuição de recursos valiosos e de serviços de saúde 50 .

As limitações deste trabalho remetem a alguns aspectos. Seu recorte transversal, em que somente associações podem ser estabelecidas e não as relações de causa e efeito, limita, até certo ponto, a interpretação dos resultados. Com exceção da variável obesidade, obtida pelas medidas físicas de peso e altura, todas as demais informações foram autorreferidas, sabendo-se que há diferenças entre as avaliações de saúde realizadas por profissionais e aquelas feitas pelos próprios indivíduos. Porém, o realce da subjetividade nas avaliações da saúde é encarado como aporte crítico essencial aos indicadores clínicos utilizados pelos profissionais de saúde, auxiliando no refinamento dos diagnósticos e na identificação de pessoas ou grupos populacionais em situação de vulnerabilidade 6,8.

A ausência de algumas variáveis na PNS 2013 fez Santos 27 adaptar seus critérios operacionais do modelo de classe original, incluindo a renda individual em substituição ao número de empregados, propriedade do estabelecimento e tamanho da propriedade agrícola, para diferenciar seis das 15 posições de classe. Essa adaptação não influenciou os resultados, porque a renda individual foi usada para distinguir as posições, mas não interferiu na definição dos agrupamentos de classe utilizados nesta análise.

As forças do presente estudo também devem ser destacadas. Primeiro, pela utilização de dados de um inquérito nacional de base populacional, com um processo de amostragem probabilística, o que possibilita generalizar mais facilmente os resultados, além de fornecer poder estatístico suficiente para estudar o desfecho em três categorias. Permite-se, com isso, analisar o gradiente escalar do estado de saúde e comparar diretamente os extremos da saúde autorreferida (positiva e negativa). Segundo, pela inclusão da classe social como um indicador robusto para o estudo dos DSS, além de sua análise estratificada segundo o sexo, fortalecendo as evidências científicas quanto às desigualdades sociais em saúde de acordo com o marcador de sexo/gênero. Por último, pela possibilidade de incluir as pessoas fora da força de trabalho como uma categoria isolada de classe social, contribuindo na discussão sobre iniquidades sociais em saúde entre os indivíduos dentro e fora do mercado de trabalho 20.

Dessa forma, os achados deste estudo reforçam a associação entre os marcadores de desigualdade em saúde e as diferenças na autoavaliação da saúde da população adulta brasileira. Mulheres, pessoas que se autodeclararam pardas e pretas, moradores das regiões Norte e Nordeste e com pior posição 
socioeconômica, aferidas com base na escolaridade, renda domiciliar per capita e classe social, tendem a apresentar pior autoavaliação da sua saúde. Sabendo-se que a autopercepção da saúde influencia a procura por serviços de saúde e a incorporação de medidas de autocuidado, esses achados podem contribuir no planejamento de políticas públicas voltadas à promoção e recuperação da saúde dos grupos sociais mais vulneráveis, impactando na redução das iniquidades que persistem no país.

\section{Colaboradores}

J. L. Sousa, G. P. Alencar, J. L. F. Antunes e Z. P. Silva participaram conjuntamente da concepção e projeto, análise e interpretação dos dados, redação do artigo, aprovação da versão final do manuscrito e possuem responsabilidade por todos os aspectos do trabalho na garantia da integralidade de qualquer parte da obra.

\section{Informações adicionais}

ORCID: Jailson Lopes de Sousa (0000-0003-18721765); Gizelton Pereira Alencar (0000-0002-23549050); José Leopoldo Ferreira Antunes (0000-00033972-9723); Zilda Pereira da Silva (0000-00034648-113X).

\section{Agradecimentos}

J. L. Sousa foi bolsista da Coordenação de Aperfeiçoamento de Pessoal de Nível Superior (CAPES - Programa Doutorado Sanduíche no Exterior processo no 88881134588/2016-01).

\section{Referências}

1. Farrer L, Marinetti C, Cavaco YK, Costongs C. Advocacy for health equity: a synthesis review. Milbank Q 2015; 93:392-437.

2. Commission on Social Determinants of Health. The social determinants of health: monitoring, research, and training. In: Commission on Social Determinants of Health, editor. Closing the gap in a generation: health equity through action on the social determinants of health. Geneva: World Health Organization; 2008. p. 178-91.

3. Wu S, Wang R, Zhao Y, Ma X, Wu M, Yan X, et al. The relationship between self-rated health and objective health status: a population-based study. BMC Public Health 2013; 13:320-9.

4. Smith-Menezes A, Duarte MFS. Fatores associados à saúde positiva autorreferida em jovens ativos na Região Nordeste, Brasil. Rev Bras Med Esporte 2013; 19:8-11.

5. Borrell C, Palència L, Muntaner C, Urquía M, Malmusi D, O'Campo P. Influence of macrosocial policies on women's health and gender inequalities in health. Epidemiol Rev 2014; 36:31-48.

6. Cebolla VM, Ascanio AL, León PDR, Barrientos RR, Castillo MJT. Desigualdad en la salud autopercibida de la población española mayor de 65 anos. Gac Sanit 2015; 28:511-21.

7. Pinillos-Franco S, García-Prieto C. The gender gap in self-rated health and education in Spain: a multilevel analysis. PLoS One 2017; 12:e0187823. 
8. Sugisawa H, Harada K, Sugihara Y, Yanagisawa S, Shinmei M. Socioeconomic status and selfrated health of Japanese people, based on age, cohort, and period. Popul Health Metr 2016; 14:27-38.

9. Barata RB, Almeida MF, Montero CV, Silva ZP. Health inequalities based on ethnicity in individuals aged 15 to 64 , Brazil, 1998. Cad Saúde Pública 2007; 23:305-13.

10. Chiavegatto Filho ADP, Laurenti R. Disparidades étnico-raciais em saúde autoavaliada: análise multinível de 2.697 indivíduos residentes em 145 municípios brasileiros. Cad Saúde Pública 2013; 29:1572-82.

11. Vincensa N, Emmelina M, Stafströma M. Social capital, income inequality and the social gradient in self-rated health in Latin America: a fixed effects analysis. Soc Sci Med 2018; 196:115-22.

12. Lorenzo T, Millán-Calenti JC, Lorenzo-López L, Sánchez A, Maseda A. Predictores de mala salud autopercibida en una población de personas mayores. Rev Esp Geriatr Gerontol 2013; 48:272-5.

13. Kong KA, Khang Y, Cho H, Jang S, Jung-Choi $K$. Neo-Marxian social class inequalities in self-rated health among the employed in South Korea: the role of material, behavioral, psychosocial, and workplace environmental factors. BMC Public Health 2017; 17:345-60.

14. Santos JAF. Classe social e desigualdade de saúde no Brasil. Rev Bras Ciênc Soc 2011; 26:27-56.

15. Buss PM, Pellegrini-Filho A. A saúde e seus determinantes sociais. Physis (Rio J.) 2007; 17:77-93.

16. Santos JAF. Uma classificação socioeconômica para o Brasil. Rev Bras Ciênc Soc 2005; 20:2746.

17. Howe LD1, Galobardes B, Matijasevich A, Gordon D, Johnston D, Onwujekwe O, et al. Measuring socio-economic position for epidemiological studies in low- and middle-income countries: a methods of measurement in epidemiology paper. Int J Epidemiol 2012; 41:871-86.

18. Barata RB, Ribeiro MCSA, Silva ZP, Antunes JLF. Classe social: conceitos e esquemas operacionais em pesquisa em saúde. Rev Saúde Pública 2013; 47:647-55.

19. Santos JAF. Classe social e deslocamentos de renda no Brasil. Dados Rev Ciênc Sociais 2015; 58:79-110.

20. Toch-Marquardt M. Does the pattern of occupational class inequalities in self-reported health depend on the choice of survey? A comparative analysis of four surveys and 35 European countries. Eur J Public Health 2017; 27 Suppl 1:34-9.

21. Damacena GN, Szwarcwald CL, Malta DC, Souza Júnior PRF, Vieira MLFP, Pereira CA, et al. O processo de desenvolvimento da Pesquisa Nacional de Saúde no Brasil, 2013. Epidemiol Serv Saúde 2015; 24:197-206.
22. Souza DE. Determinação social da saúde: associação entre sexo, escolaridade e saúde autorreferida [Tese de Doutorado]. Salvador: Instituto de Saúde Coletiva, Universidade Federal da Bahia; 2012.

23. Moor I, Spallek J, Richter M. Explaining socioeconomic inequalities in self-rated health: a systematic review of the relative contribution of material, psychosocial and behavioural factors. J Epidemiol Community Health 2017; 71:565-75

24. Moraes JR, Moreira JPL, Luiz RR. Associação entre o estado de saúde autorreferido de adultos e a área de localização do domicílio: uma análise de regressão logística ordinal usando a PNAD 2008. Ciênc Saúde Colet 2011; 16:3769-80.

25. Szwarcwald CL, Damacena GN, Souza Júnior PR, Almeida WS, Lima LT, Malta DC, et al. Determinants of self-rated health and the influence of healthy behaviors: results from the National Health Survey, 2013. Rev Bras Epidemiol 2015; 18 Suppl 2:33-44.

26. Brasil. Decreto no 7.872 , de 26 de dezembro de 2012. Regulamenta a Lei no 12.382 , de 25 de fevereiro de 2011, que dispõe sobre o valor do salário mínimo e a sua política de valorização de longo prazo. Diário Oficial da União 2012; 26 dez.

27. Santos JAF. Esquema de classe para abordar a desigualdade de saúde no Brasil. Cadernos do Itaboraí 2013; 1:39-64.

28. Rose D, O'Reilly K. The ESRC review of government social classifications. London: Office for National Statistics; 1998.

29. Instituto Brasileiro de Geografia e Estatística. Pesquisa Nacional de Saúde 2013: indicadores de saúde e mercado de trabalho. Rio de Janeiro: Instituto Brasileiro de Geografia e Estatística; 2016.

30. Instituto Brasileiro de Geografia e Estatística. Pesquisa Nacional de Saúde 2013: percepção do estado de saúde, estilos de vida e doenças crônicas. Rio de Janeiro: Instituto Brasileiro de Geografia e Estatística; 2014.

31. Landis JR, Koch GG. The measurement of observer agreement for categorical data. Biometrics 1977; 33:159-74.

32. Mortensen LH, Rehnberg J, Dahl E, Diderichsen F, Elstad JI, Martikainen P, et al. Shape of the association between income and mortality: a cohort study of Denmark, Finland, Norway and Sweden in 1995 and 2003. BMJ Open 2016; 6:e010974.

33. Malmusi D, Artazcoz L, Benach J, Borrell C. Perception or real illness? How chronic conditions contribute to gender inequalities in self-rated health. Eur J Public Health 2012; 22:781-6.

34. Pongiglione B, Stavola BL, Ploubidis GB. A systematic literature review of studies analyzing inequalities in health expectancy among the older population. PLoS One 2015; 10:e0130747. 
35. Gomes R, Nascimento EF, Araujo FC. Por que os homens buscam menos os serviços de saúde do que as mulheres? As explicações de homens com baixa escolaridade e homens com ensino superior. Cad Saúde Pública 2007; 23:565-74.

36. Cai J, Coyte PC, Zhao H. Determinants of and socio-economic disparities in self-rated health in China. Int J Equity Health 2017; 16:7.

37. Hakeberg M, Boman UW. Self-reported oral and general health in relation to socioeconomic position. BMC Public Health 2018; 18:63-71.

38. Rocha KB, Muntaner C, Solar O, Borrell C, Bernardes P, González MJ, et al. Clase social, factores de riesgo psicosocial em el trabajo y su asociación com la salud autopercebida y mental em Chile. Cad Saúde Pública 2014; 30:2219-34.

39. Supiyev A, Nurgozhin T, Zhumadilov Z, Sharman A, Marmot M, Bobak M. Levels and distribution of self-rated health in the Kazakh population: results from the Kazakhstan household health survey 2012. BMC Public Health 2014; 14:768.

40. Peres MA, Luzzi L, Peres KG, Sabbah W, Antunes JL, Do LG. Income-related inequalities in inadequate dentition over time in Australia, Brazil and USA adults. Community Dent Oral Epidemiol 2015; 43:217-25.

41. Darin-Mattsson A, Fors S, Kåreholt I. Different indicators of socioeconomic status and their relative importance as determinants of health in old age. Int J Equity Health 2017; $16: 173-84$
42. Ross C, Mirowsky J. Gender and the health benefits of education. Sociol Q 2010; 51:10.

43. Ross C, Masters R, Hummer R. Education and the gender gaps in health and mortality. Demography 2012; 49:1157-83.

44. Mackenbach JP, Kunst AE, Groenhof F, Borgan JK, Costa G, Faggiano F, et al. Socioeconomic inequalities in mortality among women and among men: an international study. Am J Public Health 1999; 89:1800-6.

45. Zajacova A. Education, gender, and mortality: does schooling have the same effect on mortality for men and women in the US? Soc Sci Med 2006; 63:2176-90.

46. Laditka J, Laditka S. Associations of educational attainment with disability and life expectancy by race and gender in the united states a longitudinal analysis of the panel study of income dynamics. J Aging Health 2016; 28:1403-25.

47. Goldthorpe JH. Analising social inequality: a critique of two recent contributions from economics and epidemiology. Eur Social Rev 2010; 26:731-44.

48. Santos JAF. A interação estrutural entre a desigualdade de raça e de gênero no Brasil. Rev Bras Ciênc Soc 2009; 24:37-61.

49. Santos JAF. Classe social, território e desigualdade de saúde no Brasil. Saúde Soc 2018; 27:556-72.

50. Viacava F, Bellido JG. Condições de saúde, acesso a serviços e fontes de pagamento, segundo inquéritos domiciliares. Ciênc Saúde Colet 2016; 21:351-70. 


\section{Abstract}

The aim of this study was to analyze self-rated health in Brazil's adult population according to markers of health inequality (color or race, region of residence, schooling, per capita household income, and social class), stratified by sex. We studied 59,758 individuals 18 years or older who participated in the 2013 National Health Survey, $a$ population-based household survey. Data collection used face-to-face interviews and key physical measurements. Self-rated health was classified as positive, fair, or negative. Multinomial logistic regression was used to estimate crude and adjusted odds ratios $(O R)$ and respective $95 \%$ confidence intervals $(95 \%$ CI). Percentage agreement and kappa values were calculated to compare the results obtained by regression models and the expected values. Prevalence of positive self-rated health in the overall population was $66.2 \%$ (70\% in men and $62.6 \%$ in women). In the adjusted analysis, the odds of worse self-rated health were significantly higher in individuals with lower per capita household income, less schooling, from the lowest social classes, residents of the North and Northeast regions, and those with brown and black color/race. Public policies for health promotion and recovery in these more vulnerable social groups can help reduce the persistent health inequalities in Brazil.

Self-assessment; Health Status Disparities; Social Conditions; Health Surveys

\section{Resumen}

El objetivo de este estudio fue analizar la autoevaluación de la salud de la población adulta brasileña, según los marcadores de desigualdad en salud (color o raza, región de residencia, escolaridad, renta domiciliaria per cápita y clase social), estratificada por sexo. Se estudiaron a 59.758 individuos, con 18 años o más de edad, que participaron en la Encuesta Nacional de Salud de 2013, una encuesta domiciliaria de base poblacional. La recogida de datos se realizó mediante entrevistas cara a cara y algunas mediciones físicas. La autoevaluación de salud se analizó como: positiva, regular y negativa. A través de una regresión logística multinomial, se estimaron odds ratio (OR) brutos $y$ ajustados y los respectivos intervalos de $95 \%$ de confianza (IC95\%). Se calcularon los porcentajes de concordancia y el valor de kappa para comparar valores obtenidos por modelos de regresión y valores esperados. La prevalencia de la autoevaluación de salud positiva en la población total fue de 66,2\%, siendo 70\% en los hombres, y 62,6\% en las mujeres. En el análisis ajustado, las oportunidades de evaluar peor la salud fueron significativamente más elevadas entre los individuos de menor renta domiciliaria per cápita, con peor nivel de escolaridad, de las clases sociales más desfavorecidas, habitantes de las regiones Norte y Nordeste y que se autodeclararon mestizos/mulatos y negros. Políticas públicas dirigidas a la promoción y a la recuperación de la salud de esos grupos sociales más vulnerables pueden impactar en la reducción de las inequidades en salud persistentes en Brasil.

Autoevaluación; Disparidades en el Estado de Salud; Condiciones Sociales; Encuestas Epidemiológicas
Recebido em 06/Dez/2018

Versão final reapresentada em 14/Out/2019 Aprovado em 21/Out/2019 